\title{
ADAPTAÇÃO CULTURAL DE UM INSTRUMENTO PARA AVALIAR AS INTERVENÇÕES DE EDUCAÇÃO TERAPÊUTICA À PESSOA COM DIABETES*
}

\author{
Laura Barbosa Nunes ${ }^{1}$, Camila Aparecida Pinheiro Landim Almeida², Malvina Thaís Pachêco \\ Rodrigues $^{3}$,Eliana Campêlo Lago ${ }^{4}$,Luana Kelle Batista Moura ${ }^{5}$, Magda Coeli Vitorino Sales Coêlho
}

Objetivo: adaptar culturalmente a Escala de Educação Terapêutica para o Comportamento do Autocuidado à Pessoa com Diabetes para o português do Brasil. Método: estudo metodológico, com análise semântica e análise de conteúdo, realizado na cidade de Teresina, Piauí, entre janeiro e julho de 2017. Foi realizada avaliação das equivalências semântica, idiomática, cultural e conceitual por oito enfermeiros. Na análise de conteúdo, foi desenvolvido um brainstorming com participação de 12 especialistas. A avaliação da pertinência prática, relevância e dimensão teórica foi feita por sete profissionais experts. Resultado: as etapas de adaptação cultural foram bem-sucedidas, e as equivalências semântico-idiomáticas, culturais e conceituais foram suficientemente alcançadas. Dos 34 itens do instrumento, 29 sofreram alterações. Obteve-se Índice de Validade de Conteúdo de 0,93 para o conjunto total de itens. Conclusão: evidenciou-se que os itens são pertinentes à cultura brasileira e avaliam aspectos na perspectiva do instrumento original, demonstrando evidências da adaptação cultural.

DESCRITORES: Educação em saúde; Diabetes mellitus; Enfermagem; Estudos de validação.

\section{CROSS-CULTURAL ADAPTATION OF A TOOL TO ASSESS THERAPEUTIC EDUCATIONAL INTERVENTIONS FOR DIABETES PATIENTS}

ABSTRACT: Objective: to identify the use of contraceptive methods by adolescents of a public school in the state of Maranhão. Method: a quantitative, descriptive-exploratory, cross-sectional study was conducted between November and December 2015, with high school adolescents from a public school in the state of Maranhão. Descriptive statistical analysis of the data was performed. Results: of the adolescents, 199 (88.1\%) reported having information on contraceptive methods, which was mainly obtained from their mothers, by $139(69.8 \%)$. Of the young women who had information, $184(92.5 \%)$ reported knowing about the male condom. Of the adolescents who had already started sexual activity, some reported not using any method, despite having the information, and $59(76.6 \%)$ young women reported having used the male condom. Conclusion: there is a need to constantly develop health actions in order to allow adolescents to exercise their sexuality more safely, so they avoid sexually transmitted infections and unplanned pregnancies.

KEYWORDS: Contraception; Adolescent; Reproductive Health; Public Health; Nursing.

\section{ADAPTACIÓN CULTURAL DE UN INSTRUMENTO PARA EVALUAR LAS INTERVENCIONES DE EDUCACIÓN TERAPÉUTICA A LA PERSONA CON DIABETES}

\begin{abstract}
Objetivo: adaptar culturalmente la Escala de Educación Terapéutica para el Comportamiento del Auto Cuidado a la Persona con Diabetes para el portugués de Brasil. Método: estudio metodológico, con análisis semántico y análisis de contenido, realizado en la ciudad de Teresina, Piauí, entre enero y julio de 2017. Se realizó evaluación de las equivalencias semántica, idiomática, cultural y conceptual por ocho enfermeros. En el análisis de contenido, se desarrolló un brainstorming con participación de 12 especialistas. La evaluación de la pertinencia práctica, relevancia y dimensión teórica se hizo por siete profesionales experts. Resultado: las etapas de adaptación cultural fueron exitosas, y las equivalencias semántico idiomáticas, culturales y conceptuales fueron suficientemente atingidas. De los 34 ítems del instrumento, 29 tuvieron alteraciones. Se obtuvo Índice de Validad de Contenido de 0,93 para el conjunto total de ítems. Conclusión: se evidenció que los ítems son pertinentes a la cultura brasileña y evalúan aspectos en la perspectiva del instrumento original, demostrando evidencias de la adaptación cultural.
\end{abstract}

DESCRITORES: Educación en salud; Diabetes mellitus; Enfermería; Estudios de validación.

\footnotetext{
*Artigo extraído do Exame de Qualificação (nível Mestrado) intitulado "Adaptação cultural de um instrumento para avaliar as intervenções de educação terapêutica a pessoa com diabetes". Programa de Mestrado Profissional em Saúde da Família. Centro Universitário UNINOVAFAPI, 2017.

'Enfermeira. Doutoranda em Enfermagem pela Universidade Federal de Minas Gerais. Belo Horizonte, MG, Brasil.

${ }^{2}$ Enfermeira. Doutora em Enfermagem. Docente Titular do Departamento de Enfermagem e do Programa de Mestrado Profissional em Saúde da Família do Centro Universitário UNINOVAFAPI. Teresina, PI, Brasil.

${ }^{3}$ Enfermeira. Doutora em Saúde Coletiva. Coordenadora do Programa de Mestrado Profissional em Saúde e Comunidade pela Universidade Federal do Piauí. Teresina, PI, Brasil.

${ }^{4}$ Enfermeira. Doutora em Biotecnologia. Coordenadora do Mestrado Profissional em Saúde da Família do Centro Universitário UNINOVAFAPI. Teresina, PI, Brasil.

${ }^{5}$ Cirurgiã-Dentista. Doutora em Endodontia. Docente do Programa de Mestrado Profissional em Saúde da Família do Centro Universitário UNINOVAFAPI. Teresina, PI, Brasil.
} 


\section{INTRODUÇÃO}

O diabetes mellitus (DM)destaca-se por sua prevalência e incidência crescentes, considerado em âmbito nacional e internacional como uma das principais síndromes de evolução crônica ${ }^{(1-2)}$.Caracterizado por hiperglicemia e ocasionado por uma deficiência absoluta e/ou relativa de insulina, o DM influencia o controle metabólico e glicêmico, podendo acarretar complicações agudas e crônicas ${ }^{(3)}$.

Embora a detecção precoce e os tratamentos adequados reduzam significativamente as mortes e complicações dessa condição crônica, ressalta-se que as intervenções baseadas em uma comunicação eficaz entre a pessoa com DM e o enfermeiro constituem uma das etapas para a adesão ao tratamento, uma vez que o profissional da saúde é capaz de promover maiores condições para comportamentos adequados de autocuidado(4-5).

Neste contexto, a educação em saúde para o autocuidado em DM é considerada a intervenção terapêutica mais direcionada, pois quando o profissional de saúde considera a pessoa com DM como o elemento principal na tomada de decisão, os cuidados tornam-se mais efetivos ${ }^{(6-8)}$. Neste contexto, aponta-se a necessidade de uma maior valorização do papel dos profissionais de saúde como sujeitos propulsores de adequações na prática educativa e no modelo de saúde ${ }^{(9-12)}$.

Frente às sucessivas dificuldades advindas para avaliar as práticas de educação para o autocuidado, surge a necessidade de adaptar culturalmente instrumentos de medida para mensurar as intervenções de educação terapêutica, visto que estas intervenções têm revelado ganhos na saúde de pessoas com DM.O presente estudo justifica-se por não terem sido identificados na literatura científica brasileira instrumentos especificamente desenhados com este propósito, já que uma correta orientação do autocuidado reduz os custos com a saúde e minimiza complicações agudas e crônicas e as incapacidades resultantes da condição crônica.

O instrumento selecionado para objeto deste estudo foi a Escala de Educação Terapêutica para o Comportamento do Autocuidado à Pessoa com Diabetes (ETCAD). Construída originalmente em Portugal, essa escala apresenta propriedades psicométricas válidas e confiáveis para avaliar o papel do enfermeiro na promoção do autocuidado nas intervenções no âmbito da educação terapêutica ${ }^{(13)}$. Trata-se de um instrumento não excessivamente longo, autoaplicável e com variados itens de fácil compreensão. Diante do exposto, o presente estudo teve como objetivo adaptar culturalmente a ETCAD para o português do Brasil.

\section{- MÉTODO}

Estudo metodológico, que visa investigar os métodos de obtenção, organização e análise de dados por meio da elaboração, validação e avaliação de instrumentos de medida ${ }^{(14)}$.

O instrumento ETACD é composto de34 itens, divididos em seis domínios (alimentação saudável, manter-se ativo, monitorização, medicação, redução de riscos, resolução de problemas e coping saudável), os quais medem a frequência de intervenções de educação terapêutica para o comportamento de autocuidado que enfermeiros da atenção básica realizam à pessoa com diabetes mellitus ${ }^{(13)}$. Cada domínio possui de dois a nove itens, e cada item apresenta uma escala do tipo Likert de cinco pontos, que varia de "sempre" a "nunca". Os escores de cada um dos seis domínios do instrumento variam de 7 a 35, 2 a 10, 5 a 25, 4 a 20, 7 a 35 e 9 e 45, respectivamente, e o escore total, de 34 a 170.

Os valores totais dos escores em cada domínio são calculados pela média aritmética simples dos itens ponderados dos respectivos domínios, e o valor total do escore da ETCAD é obtido pela média aritmética simples dos 34 itens da escala. O maior valor corresponde a uma referência de frequência alta de práticas dos enfermeiros no âmbito da educação terapêutica para comportamentos de autocuidado(13).

A autorização formal para iniciar o processo de adaptação cultural foi concedida pela autora do instrumento, Eva Patrícia da Silva Guilherme Menino. Ao considerar que a ETCAD foi construída 
originalmente em Portugal, e que Brasil e Portugal são países cuja língua nativa é o português, o processo de adaptação cultural da ETCAD foi iniciado por meio da revisão linguística por um professor de português, com nacionalidade brasileira e experiência na área da saúde, a fim de uniformizar a escrita e adequar o instrumento para o idioma da cultura-alvo, conforme a normatização do Acordo Ortográfico da Língua Portuguesa ${ }^{(15)}$. A Figura 1 ilustra o processo de adaptação cultural.

De acordo comas recomendações de Pasquali ${ }^{(16-17)}$, a versão da ETCAD uniformizada transculturalmente prosseguiu para a validação de conteúdo, conduzida sequencialmente em duas etapas: análise semântica e análise de conteúdo ou de juízes.

Durante a análise semântica, as versões da ETCAD, com os itens da escala original e os itens da versão uniformizada para o português brasileiro, foram comparadas e analisadas, no período de 1 mês, por oito juízes enfermeiros em exercício nas Unidades Básicas de Saúde (UBS). Cada juiz recebeu por correio eletrônico uma carta-convite e um material com instruções específicas para que analisassem individualmente as equivalências semântica, idiomática, cultural e conceitual da versão proposta da ETCAD. Equivalência semântica refere-se ao significado das palavras baseado na avaliação gramatical e de vocabulário. A equivalência idiomática considera as expressões equivalentes em ambos os países. $\mathrm{Na}$ equivalência cultural, as situações retratadas nos itens da versão original devem corresponder às vivenciadas na cultura-alvo. Por fim, a equivalência conceitual engloba a validade do conceito explorado ${ }^{(16-17)}$.

Posteriormente à análise semântica, procedeu-se à etapa da análise de conteúdo, com a formação do brainstorming. Conhecido por tempestade de ideias, o brainstorming é uma técnica cujo objetivo é explorar a criatividade de uma equipe, com formação de grupos variados. A técnica propõe a reunião de um maior número possível de ideias, pensamentos, experiências, visões, propostas e possibilidades que levem a um denominador comum e eficaz para a solução de problemas ${ }^{(17)}$.

Neste estudo, o brainstorming ocorreu de forma presencial em junho de 2017, em uma Instituição de Ensino Superior, em Teresina, Piauí. Nesta etapa, participaram 12 enfermeiros que apresentaram vínculo funcional formal de, no mínimo, 1ano de tempo de serviço, com as Unidades Básicas de Saúde. Assim, foram formados três grupos: quatro enfermeiros especialistas, quatro enfermeiros mestres e quatro enfermeiros doutores. Com o objetivo de facilitar a compreensão dos itens do instrumento, um integrante de cada grupo realizou a leitura individual dos itens, e foram propostas alterações de sentenças e palavras, além de sugestões e modificações, proporcionando melhor direcionamento do significado do item com o domínio associado.

Para a segunda etapa da análise de conteúdo, foram selecionados sete juízes brasileiros em agosto de 2017, por meio da consulta on-line à Plataforma Lattes, em sua maioria doutores e experts na área de adaptação e validação de instrumentos de medida em saúde. Os participantes avaliaram os itens quanto à pertinência prática, relevância teórica e dimensão teórica ${ }^{(16-18)}$. Nesta etapa, foi verificado o Índice de Validade de Conteúdo (IVC). Este índice mede a proporção ou porcentagem de juízes que estão em concordância sobre determinados aspectos do instrumento, seus itens e domínios. O método consiste em uma escala do tipo Likert com pontuação de 1 a 4 , em que $1=$ item não equivalente; $2=$ item necessita de grande revisão para ser avaliada a equivalência; $3=$ item equivalente, mas necessita de pequenas alterações; $4=$ item absolutamente equivalente ${ }^{(19)}$.

De forma a obter o cálculo do IVC, foi realizada a soma das respostas "3" e "4" dos juízes da análise de conteúdo em cada item do instrumento e dividida pela soma do número total de respostas, conforme a seguinte equação: IVC = número de respostas " 3 " ou "4" / número total de respostas. Os itens que receberam pontuações "1" ou "2" foram revisados e modificados ${ }^{(16-17,19)}$. Para que a taxa de concordância entre os participantes da análise de juízes seja considerada aceitável, o IVC deve ser superior a 0,80(15).

A pesquisa recebeu aprovação do Comitê de Ética em Pesquisa, sob o Parecer n.o 1.808 .005 , de 4 de novembro de 2016. 


\section{RESUlTADOS}

A correção ortográfica realizada pela revisora linguística resultou em adequações em quatro dos 34 itens: no item 9, "face ao nível de atividade" foi alterado para "face o nível"; no item 17, a palavra "tomas" foi substituída por "ingestões"; no item 25, "ao melhor nível possível" foi substituído por "no melhor nível possível"; e, no item 32, a expressão "a alcançar objetivos alcançáveis" foi adequada por "a atingir objetivos alcançáveis".

Dos oito enfermeiros participantes da segunda etapa da análise semântica, todos foram do sexo feminino, com média de idade de 35 anos e procedentes da região Nordeste, com 11 anos de tempo médio de formação e 9 anos de tempo médio de experiência com a temática.

Dos 34 itens, seis apresentaram sugestões de alteração da versão adaptada na primeira etapa da adaptação transcultural, o número de juízes que sugeriram mudanças na redação variou de 2 (25\%) nos itens 11, 13, 21 e 23 - a 5 (62,5\%) - no item 6. Os itens com sugestão de alteração,2, 11, 13, 21 e 23, foram avaliados com exatamente o mesmo significado nos aspectos semântico, idiomático, cultural e conceitual, porém o item 6 apresentou aproximadamente o mesmo significado nas quatro equivalências, na avaliação de 2 (25\%) juízes. A Tabela 1 apresenta a distribuição de frequências dos itens com alteração após avaliação das equivalências da escala pelos juízes.

Tabela 1 - Distribuição de frequências dos itens com alteração após avaliação das equivalências da escala pelos juízes $(n=8)$. Teresina, PI, Brasil, 2016

\begin{tabular}{lcccccccc}
\hline & \multicolumn{9}{c}{ Equivalência } \\
\cline { 2 - 8 } Item* & \multicolumn{2}{c}{ Semântica } & \multicolumn{2}{c}{ Idiomática } & \multicolumn{2}{c}{ Cultural } & \multicolumn{2}{c}{ Conceitual } \\
\cline { 2 - 8 } & AMS & EMS & AMS & EMS & AMS & EMS & AMS & EMS \\
\cline { 2 - 8 } & $\mathbf{n ( \% )}$ & $\mathbf{n ( \% )}$ & $\mathbf{n ( \% )}$ & $\mathbf{n ( \% )}$ & $\mathbf{n ( \% )}$ & $\mathbf{n ( \% )}$ & $\mathbf{n}(\%)$ & $\mathbf{n}(\%)$ \\
\hline Item 2 & - & $8(100)$ & - & $8(100)$ & - & $8(100)$ & - & $8(100)$ \\
Item 6 & $2(25)$ & $6(75)$ & $2(25)$ & $6(75)$ & $2(25)$ & $6(75)$ & $2(25)$ & $6(75)$ \\
Item 11 & - & $8(100)$ & - & $8(100)$ & - & $8(100)$ & - & $8(100)$ \\
Item 13 & - & $8(100)$ & - & $8(100)$ & - & $8(100)$ & - & $8(100)$ \\
Item 21 & - & $8(100)$ & - & $8(100)$ & - & $8(100)$ & - & $8(100)$ \\
Item 23 & - & $8(100)$ & - & $8(100)$ & - & $8(100)$ & - & $8(100)$
\end{tabular}

Legenda: AMS: aproximadamente o mesmo significado; EMS: exatamente o mesmo significado; *: versão após primeira etapa da adaptação semântica.

Nos itens 2 e 6, a expressão "hidratos de carbono" foi modificada para "carboidratos", de acordo com a sugestão de quatro $(50 \%)$ e cinco $(62,5 \%)$ juízes, respectivamente. Com relação ao item 11 , dois (25\%) juízes opinaram que "horas" fosse substituída por "horário". Nos itens 13 e 23, foi sugerida por dois (25\%) juízes a substituição de "pressão sanguínea" por "pressão arterial". E no item 21, a sugestão de dois (25\%) juízes foi a troca de "à cessação tabágica" por "ao abandono do fumo".

A análise de conteúdo, referente ao brainstorming, realizada com três estratos de enfermeiros (especialistas, mestres e doutores), totalizando 12 juízes, gerou modificações em 19 itens, conforme consta no Quadro 1. 
Quadro 1- Itens da versão adaptada culturalmente após análise semântica da validação de conteúdo ( $\mathrm{n}=12)$. Teresina, PI, Brasil, 2017

\begin{tabular}{|c|c|c|}
\hline $\mathbf{N}$ & Escala adaptada culturalmente & Versão após brainstorming \\
\hline 7 & Preparar refeições & Preparar as refeições \\
\hline 8 & $\begin{array}{l}\text { Analisar barreiras (físicas, ambientais, psicológicas e } \\
\text { de tempo) }\end{array}$ & $\begin{array}{l}\text { Identificar barreiras (físicas, ambientais, psicológicas } \\
\text { e de tempo) }\end{array}$ \\
\hline 9 & $\begin{array}{l}\text { Desenvolver um plano de atividade apropriada que } \\
\text { pondera a alimentação e medicação face ao nível de } \\
\text { atividade }\end{array}$ & $\begin{array}{l}\text { Desenvolver um plano de atividade física conside- } \\
\text { rando alimentação, medicação e nível de atividade } \\
\text { (leve, moderada e intensa) }\end{array}$ \\
\hline 12 & Interpretação e uso dos valores obtidos & Interpretação e uso dos valores glicêmicos \\
\hline 15 & $\begin{array}{l}\text { Ensinar relativamente a cada medicação: a sua ação, } \\
\text { efeitos secundários, eficácia, toxicidade. }\end{array}$ & $\begin{array}{l}\text { Ensinar relativamente a cada medicação: a sua ação, } \\
\text { efeitos adversos, eficácia, toxicidade }\end{array}$ \\
\hline 16 & $\begin{array}{l}\text { Ensinar relativamente a cada medicação: dosagem } \\
\text { prescrita, hora e frequência de administração. }\end{array}$ & $\begin{array}{l}\text { Ensinar sobre quanto a cada medicação: dosagem } \\
\text { prescrita, hora e frequência de administração }\end{array}$ \\
\hline 17 & $\begin{array}{l}\text { Ensinar relativamente a cada medicação: efeito de } \\
\text { omissão ou atraso das tomas }\end{array}$ & $\begin{array}{l}\text { Ensinar quanto à cada medicação: efeito de omissão } \\
\text { das doses ou atraso das doses }\end{array}$ \\
\hline 18 & $\begin{array}{l}\text { Ensinar relativamente a cada medicação: instruções so- } \\
\text { bre administração, armazenamento, viagens e segurança }\end{array}$ & $\begin{array}{l}\text { Ensinar relativamente a cada medicação: instruções } \\
\text { sobre segurança na administração, armazenamento, } \\
\text { transporte }\end{array}$ \\
\hline 19 & $\begin{array}{l}\text { Ensinar sobre recomendações terapêuticas com vista à } \\
\text { diminuição de riscos }\end{array}$ & $\begin{array}{l}\text { Ensinar sobre recomendações terapêuticas para mini- } \\
\text { mizar os riscos }\end{array}$ \\
\hline 21 & $\begin{array}{l}\text { Proporcionar o desenvolvimento de competências no } \\
\text { que respeita à cessação tabágica }\end{array}$ & $\begin{array}{l}\text { Proporcionar o desenvolvimento de estratégias rela- } \\
\text { tivas ao abandono do fumo }\end{array}$ \\
\hline 22 & $\begin{array}{l}\text { Proporcionar o desenvolvimento de competências no } \\
\text { que respeita à inspeção dos pés }\end{array}$ & $\begin{array}{l}\text { Proporcionar o desenvolvimento de habilidades para } \\
\text { o autocuidado de inspeção dos pés }\end{array}$ \\
\hline 23 & $\begin{array}{l}\text { Proporcionar o desenvolvimento de competências } \\
\text { no que respeita à monitorização da pressão sanguí- } \\
\text { nea }\end{array}$ & $\begin{array}{l}\text { Proporcionar o desenvolvimento de habilidades } \\
\text { relativas à monitorização da pressão arterial }\end{array}$ \\
\hline 24 & $\begin{array}{l}\text { Proporcionar o desenvolvimento de competências no } \\
\text { que respeita à automonitorização da glicemia }\end{array}$ & $\begin{array}{l}\text { Proporcionar o desenvolvimento de habilidades rela- } \\
\text { tivas à automonitorização da glicemia }\end{array}$ \\
\hline 26 & Analisar dificuldades físicas & Identificar dificuldades físicas \\
\hline 27 & Analisar dificuldades emocionais & Identificar dificuldades emocionais \\
\hline 28 & Analisar dificuldades cognitivas & Identificar dificuldades cognitivas \\
\hline 29 & Analisar dificuldades financeiras & Identificar dificuldades financeiras \\
\hline 30 & Fomentar o desenvolvimento de estratégias de coping & $\begin{array}{l}\text { Incentivar o desenvolvimento de estratégias de co- } \\
\text { ping (estilo de vida saudável) }\end{array}$ \\
\hline 33 & $\begin{array}{l}\text { Analisardificuldadesefomentarodesenvolvimentocom- } \\
\text { petênciasdecoping }\end{array}$ & $\begin{array}{l}\text { Identificar dificuldades e Incentivar o desenvolvi- } \\
\text { mento competências de coping }\end{array}$ \\
\hline
\end{tabular}

Os enfermeiros, durante o brainstorming, sugeriram retirar o item 20, o qual foi mantido para análise de conteúdo, que consiste na etapa que possibilita a exclusão de itens (a partir da análise do IVC). Foi sugerida, também, a unificação dos itens 26, 27, 28 e 29, os quais foram mantidos separadamente para avaliação na análise de conteúdo.

Na análise de conteúdo, a média de idade dos juízes foi de 32 anos, todos do sexo feminino, distribuídos entre as regiões Nordeste e Sudeste. O tempo médio de formação foi de 8 anos,e o tempo médio de experiência com a temática foi de 6 anos.

Os itens avaliados quanto àpertinência prática e relevância teórica apresentaram nas dimensões "alimentação", "manter-se ativo", "monitorização", "uso de medicação" e "redução de riscos" o IVC médio, respectivamente de 0,98,0,93,0,97,0,90 e 0,94. A última dimensão, correspondente à "resolução de problemas", apresentou menores índices médios para pertinência prática $(0,89)$ e relevância teórica $(0,82)$. Os itens foram mantidos com IVC médio aproximado de $80 \%$, correspondente a ambos os critérios. O IVC médio final desta última dimensão foi 0,86 . 
Na avaliação da dimensão teórica à qual cada item pertencia, todos foram considerados correspondentes, com concordância mínima de seis $(85,7 \%)$ juízes para "alimentação" (item 1); de seis (85,7\%) juízes para "manter-se ativo" (item 8); de cinco (71,4\%) para "monitorização" (itens 13 e 14); de sete (100\%) para as dimensões "uso de medicação" e "redução de riscos"; e concordância mínima de seis $(85,7 \%)$ juízes para "resolução de problemas" (item 26). O IVC total do instrumento, resultante da média dos índices das dimensões, foi alto $(0,93)$, bem como dos critérios avaliados de pertinência prática $(0,93)$ e relevância teórica $(0,92)$. A Tabela 2 apresenta os índices de validade de conteúdo avaliados para os critérios de pertinência prática e relevância teórica dos itens do instrumento.

Tabela 2 - Índice de Validade de Conteúdo dos itens e dimensões teóricas do instrumento segundo avaliação dos juízes da análise de conteúdo (n=7). Teresina, PI, Brasil, 2016. (continua)

\begin{tabular}{|c|c|c|c|}
\hline Item/Dimensão & $\begin{array}{l}\text { Pertinência } \\
\text { prática }\end{array}$ & $\begin{array}{l}\text { Relevância } \\
\text { teórica }\end{array}$ & $\begin{array}{l}\text { IVC } \\
\text { médio }\end{array}$ \\
\hline Alimentação & 0,98 & 0,98 & 0,98 \\
\hline 1. O efeito dos alimentos sobre a glicemia & 1 & 1 & 1 \\
\hline 2. Fontes de carboidratos, gorduras e proteínas & 1 & 1 & 1 \\
\hline 3. Fazer escolhas alimentares saudáveis & 1 & 1 & 1 \\
\hline 4. Ajustar o tamanho das porções & 1 & 1 & 1 \\
\hline 5. Ler os rótulos & 1 & 1 & 1 \\
\hline 6. Contar carboidratos & 0,86 & 0,86 & 0,86 \\
\hline 7. Preparar as refeições & 1 & 1 & 1 \\
\hline Manter-se ativo & 0,93 & 0,93 & 0,93 \\
\hline 8. Identificar barreiras (físicas, ambientais, psicológicas e de tempo) & 0,86 & 0,86 & 0,86 \\
\hline $\begin{array}{l}\text { 9. Desenvolver um plano de atividade física considerando alimentação, medicação } \\
\text { e nível de atividade (leve, moderada e intensa) }\end{array}$ & 1 & 1 & 1 \\
\hline Monitorização & 0,97 & 0,97 & 0,97 \\
\hline $\begin{array}{l}\text { 10. Instruir os pacientes sobre a escolha e seleção do equipamento de monitorização } \\
\text { da glicemia }\end{array}$ & 1 & 1 & 1 \\
\hline 11. Horário e frequência de avaliação da glicemia & 1 & 1 & 1 \\
\hline 12. Interpretação e uso dos valores glicêmicos & 1 & 1 & 1 \\
\hline 13. Ensinar a avaliar regularmente a pressão arterial & 0,86 & 0,86 & 0,86 \\
\hline 14. Ensinar a verificar regularmente o peso & 1 & 1 & 1 \\
\hline Uso de medicação & $\mathbf{0 , 9 0}$ & 0,90 & 0,90 \\
\hline $\begin{array}{l}\text { 15. Ensinar relativamente a cada medicação: a sua ação, efeitos adversos, eficácia, } \\
\text { toxicidade }\end{array}$ & 0,86 & 0,86 & 0,86 \\
\hline $\begin{array}{l}\text { 16. Ensinar sobre quanto a cada medicação: dosagem prescrita, hora e frequência } \\
\text { de administração }\end{array}$ & 0,86 & 0,86 & 0,86 \\
\hline $\begin{array}{l}\text { 17. Ensinar quanto a cada medicação: efeito de omissão das doses ou atraso das } \\
\text { doses }\end{array}$ & 0,86 & 0,86 & 0,86 \\
\hline $\begin{array}{l}\text { 18. Ensinar relativamente a cada medicação: instruções sobre segurança na } \\
\text { administração, armazenamento e transporte }\end{array}$ & 1 & 1 & 1 \\
\hline Redução de riscos & 0,94 & 0,94 & 0,94 \\
\hline 19. Ensinar sobre recomendações terapêuticas para minimizar os riscos & 0,86 & 0,86 & 0,86 \\
\hline 20. Estabelecer objetivos com vista à diminuição de riscos & 0,86 & 0,86 & 0,86 \\
\hline 21. Proporcionar o desenvolvimento de estratégias relativas ao abandono do fumo & 1 & 1 & 1 \\
\hline $\begin{array}{l}\text { 22. Proporcionar o desenvolvimento de habilidades para o autocuidado de ins- } \\
\text { peção dos pés }\end{array}$ & 1 & 1 & 1 \\
\hline
\end{tabular}




\begin{tabular}{|c|c|c|c|}
\hline $\begin{array}{l}\text { 23. Proporcionar o desenvolvimento de habilidades relativas à monitorização } \\
\text { da pressão arterial }\end{array}$ & 1 & 1 & 1 \\
\hline $\begin{array}{l}\text { 24. Proporcionar o desenvolvimento de habilidades relativas à automonitorização } \\
\text { da glicemia }\end{array}$ & 1 & 1 & 1 \\
\hline $\begin{array}{l}\text { 25.Proporcionar o desenvolvimento de competências no que respeita à } \\
\text { manutenção dos cuidados pessoais no melhor nível possível }\end{array}$ & 0,86 & 0,86 & 0,86 \\
\hline Resolução de problemas & 0,89 & 0,82 & 0,86 \\
\hline 26. Identificar dificuldades físicas & 0,86 & 0,71 & $\mathbf{0 , 8 0}$ \\
\hline 27. Identificar dificuldades emocionais & 0,86 & 0,71 & 0,80 \\
\hline 28. Identificar dificuldades cognitivas & 0,86 & 0,71 & 0,80 \\
\hline 29. Identificar dificuldades financeiras & 0,86 & 0,71 & 0,80 \\
\hline $\begin{array}{l}\text { 30. Incentivar o desenvolvimento de estratégias de coping (estilo de vida saudá- } \\
\text { vel) }\end{array}$ & 1,00 & 1,00 & 1,00 \\
\hline 31. Identificar a motivação do paciente para a mudança comportamental & 1 & 1 & 1 \\
\hline 32. Ajudar o paciente a atingir objetivos alcançáveis & 0,86 & 0,86 & 0,86 \\
\hline 33.Analisar dificuldades e fomentar o desenvolvimento competências de coping & 0,86 & 0,86 & 0,86 \\
\hline 34. Avaliar os pacientes quanto ao seu estado emocional & 0,86 & 0,86 & 0,86 \\
\hline IVC total & 0,93 & 0,92 & 0,93 \\
\hline
\end{tabular}

Legenda: IVC: Índice de Validade de Conteúdo.

\section{DISCUSSÃO}

O desenvolvimento do processo de adaptação cultural da ETCAD para o uso no Brasil foi realizado de forma sistematizada e foi conduzido conforme as recomendações preconizadas nos estudos de Pasquali para adaptação cultural de instrumentos de medida ${ }^{(15-16)}$. A utilização de normas metodológicas internacionalmente aceitas para a realização de adaptação cultural facilita a reprodutibilidade dos resultados e possibilita a comparação entre diferentes populações ${ }^{(13,20)}$.

A revisão linguística foi realizada sem variações do vocabulário ortográfico português, conforme as normas do Novo Acordo Ortográfico da Língua Portuguesa. Logo, apenas quatro itens foram modificados, e o significado de cada item do instrumento foi mantido.

Neste estudo, o processo de adaptação foi realizado com a participação enfermeiros especialistas na área de adaptação e validação de instrumentos e/ou atuação prática no autocuidado da pessoa com diabetes mellitus. A composição de enfermeiros especialistas, mestres e doutores, com experiência na temática, permitiu um processo de adaptação amplo e profundo, com observações pertinentes e complementares $^{(21)}$.

Durante a avaliação das equivalências semânticas, idiomáticas, conceituais e culturais, realizada por oito juízes (especialistas e mestres),foram realizadas alterações em seis itens do instrumento, ou seja, algumas palavras ou expressões foram substituídas a fim de facilitar a compreensão, pois, apesar da escrita uniformizada entre Portugal e Brasil, há peculiaridades culturais, sendo utilizadas palavras iguais para expressar objetos diferentes ou palavras que não são utilizadas no outro país ${ }^{(20)}$.

Posteriormente, com vistas a verificar se todos os itens foram compreensíveis para todos os membros da população aos quais se destinam, realizou-se a análise de juízes ou análise de conteúdo, baseada no julgamento realizado por um grupo de juízes experientes, aos quais cabe analisar se o conteúdo está adequado ao que se propõe ${ }^{(21)}$. Um estudo de validade de conteúdo pode fornecer informações sobre a representatividade e clareza de cada item com a colaboração de especialistas, porém existem limitações nos estudos de validade de conteúdo que precisam ser observadas, visto que a análise dos especialistas é subjetiva e, por conseguinte, podem existir distorções nos estudos ${ }^{(22)}$. 
O método do brainstorming utilizado nesta pesquisa mostrou-se eficaz na avaliação da compreensão dos itens. Entretanto, surgiram divergências na reprodução de 19itens da ETCAD, pois o comitê de juízes afirmou possuir entendimento diferente do que se busca compreender, dificultando a orientação para a população à qual se destina, assim como afirma o estudo de Pasquali ${ }^{(17)}$. Também houve a sugestão para a unificação de quatro itens(identificar dificuldades físicas, emocionais, cognitivas e financeira), porém estes foram mantidos separados, uma vez que a resposta de um pode ser atribuída aos quatro.

Pesquisa destaca que uma adaptação adequada da equivalência cultural é de fundamental importância para que os termos utilizados no instrumento sejam coerentes com a realidade vivenciada pela população-alvo, dentro de seu contexto cultural. Caso o teor cultural se encontre fora do contexto ou da vivência daquela população, deve ser modificado ${ }^{(23)}$.

Durante o procedimento de adaptação cultural da ETCAD, foi possível realizar alterações que respeitaram as particularidades da população-alvo do instrumento, com o intuito de que este seja bem compreendido. A ETCAD foi traduzida, adaptada e apresentou valores satisfatórios de validade de conteúdo, baseados nos altíssimos valores das médias dos índices das dimensões ou domínios, bem como pertinência prática e relevância teórica.

Verificou-se que as etapas de adaptação cultural foram bem-sucedidas, e as equivalências semânticoidiomáticas, culturais e conceituais foram obtidas. Os juízes experts concluíram que os conceitos do instrumento são pertinentes à cultura brasileira, e os itens são relevantes aos domínios avaliados pelo instrumento. Dessa forma, o título do instrumento foi mantido para a versão brasileira: "Escala de Educação Terapêutica para o Comportamento do Autocuidado à Pessoa com Diabetes", além disso, não houve eliminação de nenhum dos itens do instrumento original, apenas modificações de expressões ou inversão de palavras para facilitar o entendimento.

Os resultados demonstram que a versão adaptada do instrumento foi bem compreendida e adequada para a verificação de suas propriedades psicométricas na população-alvo. A versão brasileira da ETCAD encontra-se em processo de avaliação das propriedades psicométricas. Depois da finalização do processo, o instrumento estará disponível para ser utilizado nas unidades básicas de saúde no Brasil.

Assim, a utilização dessa escala pelos profissionais possibilitará a identificação das práticas de educação terapêutica, com ênfase nos comportamentos de autocuidado, desenvolvidas pelos enfermeiros, para posteriormente identificar o contributo dos enfermeiros para os ganhos em saúde. Entretanto, torna-se necessária a monitorização dessas intervenções, no sentido da análise da qualidade, ajuste dos cuidados e revisão de metas a serem alcançadas.

\section{CONCLUSÃO}

A adaptação transcultural e a validação de conteúdo da ETACD demonstraram que os itens do instrumento adaptado são pertinentes à cultura brasileira e avaliam aspectos na perspectiva do instrumento original. Portanto, a adaptação cultural da ETCAD possibilita contribuir para a produção do conhecimento científico de estudos metodológicos em diferentes contextos culturais, permitindo a adaptação semântica e validação.

Diante dos resultados desta pesquisa, novos estudos estão sendo realizados para a avaliação dos parâmetros psicométricos do instrumento adaptado, a fim de viabilizar a sua utilização no Brasil, tendo em vista a importância de avaliar as intervenções terapêuticas para a educação em diabetes, abrindo portas para a parceria entre ensino e serviço, com o intuito de melhorar a autogestão da pessoa com essa importante condição crônica de saúde.

É importante articular as práticas de educação para o autocuidado da pessoa com diabetes mellitus, desenvolvidas por enfermeiros, para que tais práticas sejam adequadas, no sentido do alcance de melhores controles metabólicos. Espera-se que os indivíduos com DM adquiram hábitos de boas práticas de autocuidado e sejam acompanhados continuamente por enfermeiros e profissionais de saúde, evitando e minimizando complicações agudas e crônicas associadas, as quais interferem nas condições e qualidade de vida dessa população. 


\section{○ REFERÊNCIAS}

1. International Diabetes Federation (IDF) Diabetes Atlas7. ed. Brussels: IDF. [Internet] 2015 [acessoem 8 jul 2017]. Disponível: https://www.idf.org/sites/default/files/EN_6E_Atlas_Full_0.pdf

2. Sociedade Brasileira de Diabetes (SBD). Diretrizes da Sociedade Brasileira de Diabetes (2015-2016). São Paulo: A.

C. Farmacêutica; 2016.

3. American Diabetes Association (ADA). Diagnosis and classification of diabetes mellitus. American Diabetes Association. [Internet] 2016:37(Suppl 1) [acesso em 12 jul 2017]. Disponível: https://doi.org/10.2337/dc14-S081.

4. Borba AKOT, Marques APO, Leal MCC, Ramos RSPS. Práticas educativas em diabetes Mellitus: revisão integrativa da literatura. Rev Gaúcha Enferm.. [Internet] 2012;33(1) [acesso em 10 mai 2017]. Disponível: http://dx.doi.org/10.1590/ S1983-14472012000100022.

5. de Melo LP, de Campos EA. "O grupo facilita tudo": significados atribuídos por pacientes portadores de diabetes mellitus tipo 2 a grupos de educação em saúde. Rev. Latino-Am. Enfermagem. [Internet]2014;22(6) [acesso em 8 jul 2017]. Disponível: http://dx.doi.org/10.1590/0104-1169.0056.2506.

6. Almeida CAPL, da Cruz JN, Fernandes DR, Silva LQ, de Lima CHR, Lago EC. Nurse training in primary care: educational actions with people living with diabetes mellitus. Acta Scientinarum. Health Sciences. [Internet] 2017;39(2) [acesso em 10 set 2017]. Disponível: http://dx.doi.org/10.4025/actascihealthsci.v39i2.33389.

7. Torres HC, Pereira FRL, Alexandre LR. Avaliação das ações educativas na promoção do autogerenciamento dos cuidados em diabetes mellitus tipo 2.Rev. esc. enferm. USP. [Internet] 2012;45(5) [acesso em 3 ago 2017]. Disponível: http://dx.doi.org/10.1590/S0080-62342011000500007.

8. Mendonça FF, Nunes EFPA. Avaliação de grupos de educação em saúde para pessoas com doenças crônicas.Trab. educ. saúde. [Internet] 2015;13(2) [acesso em 23 ago 2017]. Disponível: http://dx.doi.org/10.1590/1981-7746-sip00053.

9. Ministério da Saúde (BR). Secretaria de Atenção à Saúde. Departamento de Atenção Básica. Estratégias para o cuidado da pessoa com doenças crônicas: diabetes mellitus. [Internet] Brasília: Ministério da Saúde; 2013 [acesso em 3 ago 2017]. Disponível:http://189.28.128.100/dab/docs/portaldab/publicacoes/caderno_36.pdf.

10. Imazu MFM, Faria BN, de Arruda GO, Sales CA, Marcon SS. Effectiveness of individual and group interventions for people with type 2 diabetes. Rev. Latino-Am. Enfermagem. [Internet] 2015;23(2) [acesso em 10 jul 2017]. Disponível: http://dx.doi.org/10.1590/0104-1169.0247.2543.

11. Hass L, Maryniuk M, Beck J, Cox CE, Duker P, Fisher EB et al. National standards for diabetes self-management education and support. Diabetes Care. [Internet] 2012;35(11) [acesso em 11 ago 2017]. Disponível:https://doi.org/10.2337/ dc12-1707.

12. Klein HA, Jackson SM, Street K, Whitacre JC, Klein G. Diabetes self-management education: miles to go. Nurs Res Pract. [Internet] 2013;2013 [acesso em 24 jul 2017]. Disponível: http://dx.doi.org/10.1155/2013/581012.

13. Menino EPSG, Dixe MACR, Louro MCCM. Construção e validação da escala de educação terapêutica para o comportamento de autocuidado na diabetes. Revista de Enfermagem Referência. [Internet] 2016;4(8) [acesso em 24 ago 2017]. Disponível: http://dx.doi.org/10.12707/RIV15049.

14. Polit DF, Beck CT. Fundamentos da pesquisa em enfermagem: avaliação de evidências na prática de enfermagem.7 ed. Porto Alegre: Artmed; 2011.

15. Ministério da Educação (BR). Acordo ortográfico da língua portuguesa: atos internacionais e normas correlatas. [Internet] Brasília: Coordenação de Edições Técnicas; 2014 [acesso em 19 jul 2017]. Disponível: https://www2.senado. leg.br/bdsf/bitstream/handle/id/508145/000997415.pdf?sequence=1 . 
16. Pasquali L. Instrumentos psicológicos: manual prático de elaboração. Brasília: LabPAM/IBAPP; 1999.

17. Pasquali L. Instrumentação psicológica: fundamentos e práticas. Porto Alegre: Artmed; 2010.

18. Medeiros RKSM, Ferreira Junior MA, Pinto DPSR, Vitor AF, Santos VEP, Barichello E. Modelo de validação de conteúdo de Pasquali nas pesquisas em enfermagem. Revista de Enfermagem Referência. [Internet] 2015;4(4) [acesso em 19 jul 2017]. Disponível: http://dx.doi.org/10.12707/RIV14009.

19. Alexandre NMC, Coluci MZO. Validade de conteúdo nos processos de construção e adaptação de instrumentos de medidas. Ciênc saúde coletiva. [Internet] 2011;16(7) [acesso em 22 mai 2017]. Disponível: http://dx.doi.org/10.1590/ S1413-81232011000800006.

20. Galdeano LE, Furuya RK, Delacio MCB, Dantas RAS, Rossi LA. Validação semântica do CardiacPatients Learning NeedsInventorypara brasileiros e portugueses. Rev Gaúcha Enferm. [Internet] 2011;32(3) [acesso em 23 ago 2017]. Disponível: http://dx.doi.org/10.1590/S1983-14472011000300024.

21. de Mendonça SCB, Zanetti ML, Sawada NO, Barreto IDC, de Andrade JS, Otero LM. Construction and validation of the Self-care Assessment Instrument for patients with type 2 diabetes mellitus. Rev. Latino-Am. Enfermagem. [Internet] 2017;25 [acesso em 15 agosto 2017]. Disponível: http://dx.doi.org/10.1590/1518-8345.1533.2890.

22. Torres HC, Chaves FF, daSilva DDR, Bosco AA, Gabriel BD, Reis IA, et al. Translation, adaptation and validation the contents of the Diabetes Medical Management Plan for the Brazilian context. Rev. Latino-Am. Enfermagem. [Internet] 2016;24 [acesso em 22ago2017]. Disponível: http://dx.doi.org/10.1590/1518-8345.1138.2740.

23. Van der Heide I, Uiters E, Rademakers J, Struijs JN, Schuit AJ, Baan CA.Associations among health literacy, diabetes knowledge, and self-management behavior in adults with diabetes: results of a dutch cross-sectional study.J Health Commun.[Internet]2014;19(Suppl2) [acesso em 26ago2017]. Disponível: http://dx.doi.org/10.1080/10810730.2014.9 36989. 